\title{
Human Resource Management: Pre-pandemic, Pandemic and Beyond
}

\author{
Binoy Mathew $\mathrm{KV}^{1 *}$ and Maryelizabeth Tidiya Walarine ${ }^{2}$ \\ 'Department of Physiotherapy, Composite Regional Centre for Skill Development Rehabilitation \\ and Empowerment of Persons with Disabilities, Kozhikode, Kerala, India \\ https://orcid.org/0000-0002-9637-1214 \\ 2Department of Medical Surgical Nursing, MIMS College of Nursing, Malappuram, India \\ https://orcid.org/0000-0003-4994-2229 \\ *Email Correspondence: binoy9797@gmail.com
}

\begin{abstract}
The pandemic disrupts the functioning of most sectors and exposes the shortcomings and vulnerabilities among many. Literature search in leading databases reveals that though shocked by the crisis, many industries and organizations have reinvented themselves through various strategies to overcome the consequences of the pandemic. After a thorough analysis of individual articles, the authors found out that human resource management considers the disruptive changes in the working pattern, challenges, and opportunities ahead and makes appropriate changes to create an efficient workforce for the future with the help of technology that has become an inevitable companion in human resource management and not a panacea for uncertainties and crises ahead.
\end{abstract}

Keywords: human resource management, pandemic, HRM, new normal, strategies

\subsection{Introduction}

COVID-19 pandemic has emitted shockwaves, disrupting the functioning of most sectors across the globe. The pandemic exposed the vulnerabilities, shortcomings, and resource scarcities of the sectors. Imbalances and inequalities thus created by the pandemic will prevail for an extended period. And human resources across the world are not equipped to handle the pandemic and its consequences. Lockdowns and associated travel restrictions disrupted work, as well as working patterns, of many sectors. Uncertainty, the unpredictability of the future, and concerns regarding getting infected had adversely affected the psycho-emotional health of the workers.
Human Resource Management (HRM) is defined as 'all those activities associated with the management of employment relations in the firm, and human resource is described as "all the knowledge, skills, networks, and energies of people and, underpinning them, their physical and emotional health, intellectual capabilities, personalities, and motivations (Armstrong \& Taylor, 2014). Undeniably, the current global crisis brings forth the need to devise and develop strategies to anticipate and respond effectively to many adverse situations and problems in various forms. And the human resource management cannot stand alone and be static in the scenario. 
A 4-R Crisis-Normalcy Model comprising the stages Redefine, Relook, Redesign, and Reincorporate proposed guiding the organizations through the pandemic and beyond (Arora \& Suri, 2020). The 'Redefine' stage suggests a critical analysis of the existing HRM, operational processes, strategies, and optimum modifications by keeping the employee experiences in view. Following it is the transition stage called 'Relook,' where the necessity to make changes is communicated to employees using various communication modes. The modifications needed in an organization will be planned and skill up-gradation avenues for achieving it will be explored. 'Redesign' is the stage of implementing the changes in the organization with employee support by collaborating with the stakeholders. In the final stage, 'Reincorporating,' the changes will be completed and additional measures will be implemented to enhance flexibility and productivity (Arora \& Suri, 2020). Human resource management strategies adopted in the new normal will determine the secured future and safe existence of the workforce and, thereby, humanity.

\subsection{Design and Methods}

The present study was a narrative review to understand the human resource management strategies used in the pre-pandemic and pandemic periods and recommend strategies that can be used in the future. The study was performed based on the Preferred Reporting Items for Systematic Reviews and Meta-analyses guidelines (Moher et al., 2009).

\section{Search Strategy}

An extensive literature search was conducted in databases Wiley, ScienceDirect, PubMed, Sagepub with keywords COVID-19, Pandemic, Human
Resource, Management, Workforce, Strategies, and Measures. A search was conducted in online newspapers and management websites with various combinations of the keywords mentioned above. Only articles that discussed pandemicrelated human resource management strategies and published in English between January 2020 to October 2021 were considered.

\section{Selection of Articles and Document}

The authors of this paper independently screened the abstracts and titles for eligibility. After a thorough study and when the reviewer found the abstract or title potentially useful, full copies of the articles were then retrieved and considered for eligibility. The authors conducted frequent meetings to reach a consensus regarding the eligibility of doubtful articles and documents.

\section{Inclusion and Exclusion Criteria}

The inclusion and exclusion criteria are (a) being published from January 2020 to October 2021, in English, and a scientific research journal, an online newspaper, or management website; and (b) examining human resource management strategies related to pandemic.

\section{Database Search}

The initial database search of the literature resulted in a total of 2312 documents. In the next step, duplicated articles, books, dissertations, and presentations were excluded, and the number of documents decreased to 546 . Based on systematic screening in the first stage, the authors reviewed the title and abstracts to find those related to human resource management during the pandemic and extracted 64 eligible documents. In the next step, all 64 full-texts were considered and finally, 26 of them were selected. 


\subsection{Discussion}

\section{Human Resource Management Strategies Adopted in the Pre-pandemic Period}

During the pre-pandemic period, Human Resource Management was done mostly onsite. Recruitment, training, and performance management were done physically with a oneto-one contact in the workplace. Job design and work supervision designed for onsite physical meetings were conducted among team members, with superiors, clients, and other stakeholders. Remote working was rarely practiced during the pre-pandemic period.

\section{Human Resource Management Strategies Adopted in the current pandemic}

The pandemic opened opportunities for Human Resource (HR) managers and faculty members to introspect, reflect on existing HRM practices, and explore reformative, innovative, and "Out of the Box" solutions to the challenges ahead. Strategic thinking encourages HR managers to view the pandemic differently and act appropriately as catalysts who stimulate wise realizations and induce an array of challenges and opportunities. The business world, work culture, and working patterns will not be the same in the new normal. So human resource needs to be wellprepared to address the challenges and explore the opportunities of the post-pandemic era. In the future competitive world, easy adaptability, agility, good communication, excellent coordination, responsible leadership, and appropriate technology application are the traits that need to be developed by human resources (Margherita \& Heikkila, 2021).

In some industries where remote working or "work from home" was possible, the sudden surge in such a work pattern challenged the existing technical infrastructure. Initially, sectors were skeptical regarding the efficacy of remote working, but gradually with training programs, schedule creation, and infrastructure up-gradation, they overcame it (Howe et al., 2020). Microsoft prepared a guideline titled 'Guide to Working from Home during COVID-19' and circulated it among its employees. An editable version of the document was shared in the public domain so that other institutions could modify it according to their needs (Microsoft, 2020). Besides, Walmart and Best Western Hotel started utilizing virtual reality services for employee training (Meister, 2020). Remote working results in extensive cost savings for organizations by reducing the cost of office spaces, utilities, and travel expenses. From an employee's perspective, remote working helps save travel time and related expenses, thereby, paving the way to sufficient time for personal needs and other commitments.

Concerned with their employees' psycho-social well-being, many companies took appropriate measures. For instance, Takeda, a Japanese company, created a virtual platform named "Desk Yogi" to assist their employees' general well-being and address stress management issues. Another example is Sprinklr, an American company, which created a smartphone application called "Headspace" to provide its employees a guided meditation (Aurelia \&Momin, 2020). Clix Capital in India also prepared a platform to promote the mental well-being of its employees (Arora \& Suri, 2020).

Cross-industry temporary sharing of employees can be mutually beneficial for the industries. It was successfully implemented in China during the pandemic period (Cooke et al., 
2020). Temporary re-routing the employees to other industries where the service demand is high helps tackle the majority of the issues faced by employees and the present employer. This arrangement provides a steady income for the employees, ensures the needed workforce for the industry with service demand, and relieves the present employer from providing compensatory relief measures during unemployment.

The hospitality industry adopted pay reduction and slashing of incentives as human resource management strategies during the pandemic. In India, for example, employees working in restaurants, bars, salons, and multiplexes faced pay reductions and were given leave without pay during the pandemic (Bhusan \& Verma, 2021). Casinos in Las Vegas also adopted layoffs and furloughs to address the pandemic-related crisis (DeLeon \& Geller, 2020). These were made possible as good communication and warm behavior from the hospitality sector made the employees less fearful of facing uncertainties (Agarwal, 2021). Another recovery strategy was to focus more on domestic travelers. Moreover, the pandemic has influenced consumer interests and expenditure patterns owing to health and safety concerns. Considering this, the hospitality industry organized online training programs which equip employees with healthy conduct appropriate during the pandemic. Preference for low-cost alternatives like rented cars, domestic tourism, and short-stay home rentals was also on a hike (Pappas \& Glyptou, 2021).

Countries that adopted systematic thinking and practices faced the uncertainty caused by the pandemic effectively. Women leaders successfully controlled the pandemic in their countries as they adopted scientific and evidence-based approaches with empathetic minds (McLean \& Jiantreerangkoo,
2020). These systematic thinking strategies can be adopted by HR managers, too.

\section{Human Resource Management Strategies to be Adopted in the Future}

Various Human Resource Management (HRM) strategies can be adapted to face the uncertainties and challenges in the future. Due to the rapid changes in demands, uncertainties, and challenges in multiple dimensions, organizations will be forced to upgrade themselves into learning organizations. Team learning, shared vision, personal mastery, mental models, and systems thinking are Peter Senge's foundations of learning organizations (Altındağ \& Bilaloğlu Aktürk, 2020; Senge, 1997). According to Peter Senge, people should put aside their old ways of thinking (mental models), learn to be open with others (personal mastery), understand how their organization works (systems thinking), form a plan everyone can agree on (shared vision), and then work together to achieve that vision (team learning) (Senge, 1997). Innovative and proactive strategies should be practiced to produce needed changes in such organizations. Moreover, lessons can be learned by retrospectively analyzing how industries transformed themselves successfully after stagnation during World War II. Innovation, product diversification, skills training, employee welfare schemes, and finding solutions to new challenges were the strategies used during that time (Rondi et al., 2021). Hence, human resource managers undoubtedly play a crucial role in making the work climate conducive to such changes.

\section{Job Design}

The successful HRM strategies used by familyowned business enterprises such as less formal employee management, broad job description, flat hierarchy, and more responsibilities at an 
earlier age can be adopted by organizations in the new normal (Rondi et al., 2021). Instead of job specialization, job generalization can become a norm that enables employees to handle multiple job roles (Hite \& McDonald, 2020). Such HRM strategies can help industries face the postpandemic challenges and overcome them.

\section{Training and Development}

Training and development are key HRM strategies for addressing the challenges in the post-pandemic era. Many companies invest in research, development, and innovation. They either devise new products or services or modify and discard existing products or services. To achieve all these, human resources need to be nurtured and developed accordingly.

Inclusive Human Resource Management is given due emphasis in recent times. Inclusion is defined as "a culture that connects each employee to the organization; encourages collaboration, flexibility, and fairness; and leverages diversity throughout the organization so that all individuals can participate and contribute to their full potential" (Shore et al., 2018). According to Society for Human Resource Management (SHRM), inclusion is a two-way accountability wherein each person must grant and receive inclusion from others (Society for Human Resource Management, 2021). Inclusive HRM ensures a smooth transition from training student to employee, unemployed to employed, or shifting between jobs. It was found that if the unemployed are provided adequate training with new skill sets, they will be empowered to secure jobs (Borghouts - van de Pas et al., 2021). However, employees working with international organizations and clients are forced to work long hours to accommodate the time zone differences. This work condition can cause physical and mental stress on the employees. As a remedy, asynchronous and synchronous virtual meetings can be employed to spare employees from stress. Furthermore, virtual collaborations and virtual leadership can also be enhanced in the post-pandemic era. Besides working in a virtual environment crosses national boundaries and brings together employees from diverse cultures. It necessitates virtual-based cross-cultural training for such employees to have efficient virtual collaborations and avoid possible conflicts. Consequently, future HR managers should be adequately trained to be efficient virtual leaders.

The United Nations Sustainable Development Goals (SDGs) emphasize the need for Good Health andWellbeing. To achieve this, strengthening health systems across the world becomes a necessity. The pandemic had shown the glaring inadequacy and fragility of the health systems in many countries (Tonne, 2021). Human resource development in the healthcare sector, along with the underlying infrastructure and its effective management, can be a solution. Intensive training on telemedicine and task shifting should be conducted among the human resources in the healthcare sector (TanTorres Edejer et al., 2020).

\section{Technology Adoption and Implementation}

Embracing appropriate and beneficial technologies that are currently available is necessary for succeeding in the new era. Organizations are forced to digitalize partially or completely their strategic and operational functions to keep abreast of digital updates and transformation. Moreover, virtual assistants help companies provide uninterrupted round-the-clock customer services. Cloud-based systems, Artificial Intelligence (AI), Internet of Things (IoT), sensor technologies, and robotics will also be extensively 
used in the upcoming years (Aurelia \& Momin, 2020; Fachrunnisa \& Hussain, 2020). All these call for close collaboration between the industrial sector and the related technical training institutes.

The recruitment process in the future will mostly be online by utilizing machine learning and Artificial Intelligence (Al) (Aurelia \& Momin, 2020). The online process helps reduce recruitmentrelated costs and travel expenses for both employers and candidates. Utilizing an online platform for application submission, verification, shortlisting, screening examinations, and videoconferencing interviews become a common practice. Such strategic human resource management helps align the long-term objectives of various industries to deliver high-quality services and products. Artificial Intelligence aids in the facilitation of more customer-friendly, value-based services and also helps in problem-solving. In addition, blockchain technology helps identify the core skill sets to be possessed by future employees in each sector and supports the design curriculum and employee training such that they inculcate these skills in the prospective and existing workforce (Fachrunnisa \& Hussain, 2020). With the advent of smartphonebased services that are readily accessible in many small and middle-level enterprises across the world, mobile-based business communications and payment pave the way to the sustainability of many industries. Thus, the human workforce needs to be trained in smartphone-based communications and payment transactions for this new world.

As the pandemic exposed the fragility of the distribution system and consequences of relying heavily on imports, national as well as regional level self-reliance are given more thrust. This condition leads to new employment opportunities and exerts pressure to adopt advanced technologies for manufacturing, marketing, and logistics. Companies embracing technology is not the only panacea for post-pandemic challenges but also organizations following ethical and employeefriendly HRM principles.

\section{Innovation and Creativity}

Innovation is considered the powerful key in opportunities and organizational resilience, especially in the manufacturing and service sectors. It can be product innovation, management system innovation, or process innovation (CaballeroMorales, 2021). Creating innovative solutions to the new and anticipated problems by reinventing the existing infrastructure and human resources is recommended to overcome the current crisis gripping these sectors (Kuckertz et al., 2020). The approaches which help in this process are the DMAIC (Define, Measure, Analyze, Improve and Control) model, Michael Porter's Five Forces, and the Blue Ocean strategy. Michael Porter's Five Forces are the threat of entry, the intensity of competition, the threat of substitutes, power relative to buyers and suppliers while the Blue Ocean strategy involves the creation of new market space by creating demand for a new product (Caballero-Morales, 2021).

The key determinants for the economic advancement of countries are innovation, knowledge generation, high-skilled labor, research, and development (Shin et al., 2020). The states and geographical units should develop a long-term strategic plan to become self-reliant in meeting their basic health, food, and service needs. Mobility and travel restriction can repeat at any time, owing to increasing man-made, biological, sociopolitical, and natural calamities. Cross-pollination of ideas from various industries especially the health sciences is highly encouraged 
during the post-pandemic period because it is the health sector that is primarily confronted by the invisible enemy amidst the uncertainties, vulnerabilities, and resource limitations. In addition, interdisciplinary insight sharing leads to the innovation and development of novel applications (Shin et al., 2020). So, HR professionals should enhance their tolerance for uncertainty and face challenging situations proactively and optimistically. Technology then should be considered for assistance in HRD activities and not as a replacement for HR professionals.

\section{Embracing Paradigm Shift}

The industries like entertainment, travel, and tourism need to relearn ways to stay profitable in the new normal. In product development industries, the demand is not the same as it was in the pre-pandemic period. So, modification of the existing products or redirection to new product development becomes mandatory. Many industries may not be able to afford to maintain the prepandemic era strategies but they can adopt leaner organizations. For instance, the unskilled or lowlevel skilled employees who will be facing tough competition in the post-pandemic period will be greatly affected if the industries plan to shift to a leaner structure. Therefore, training and securing new skills is the way forward. Nevertheless, some researches suggest retaining the pre-pandemic level of the workforce and focusing on human resource development to meet future challenges. Such an approach will certainly create loyal and committed employees (Howe et al., 2020).

Hospitality and tourism businesses can only overcome the current crisis if they make strategic changes in their operations and approaches and give utmost consideration to customers' health, hygiene, infection risk avoidance, and cost affordability. The local government can also take adequate measures to make the tourism destinations safe and healthy. To add, attractive and affordable costs and services help to lure domestic tourists. Besides, online food delivery is coming up as a business alternative in urban settings. And to keep abreast in the post-pandemic period, health and well-being services should be integrated into the service portfolio (Le \& Phi, 2021). Needless to say, human resources should be equipped with the skills and commitment to handle these transformations.

In the Project-Based Organizations (PBO), employees are assigned specific tasks during a project period. Simultaneously, the same employees perform other different specific tasks related to various projects. While the HRM practices and processes are different from a permanent organizational setting (Samimi \& Sydow, 2021), $\mathrm{PBO}$ will be extensively used in the post-pandemic period. Thus, human resources need to be trained to meet the unique challenges and opportunities offered by these temporary organizations.

\section{Remote Working}

Remote working or Work From Home(WFH) became the norm in the new normal in many sectors and business settings. Many organizations were unexposed to this work pattern and unprepared for the human resource management of remote working. Digital monitoring was used by many companies for remote work to measure employee job performance virtually. Participants of a qualitative study shared the view that monitoring helped them in coping with procrastination of work (Wang et al., 2021). In the Information Technology sector, some organizations helped their employees by providing infrastructural support development assistance for ensuring remote work productivity 
(Shankar, 2020). Employers should consider the work characteristics of their employees considered as they design remote working policies. Employee well-being and work-home balance should also be given premium when devise remote workingrelated HRM policies (Mathew K V \& Walarine, 2021). Provision for social support, not just communication of company updates, through online gathering can address the negative effects of loneliness, enhance emotional well-being and the sense of belonging (Bavik et al., 2020).

\section{Hybrid Working Strategies}

Hybrid working involves a combination of remote working and onsite working. Nature of work, the personality of the employees, level of experience, and age are factors that determine the selection of hybrid working arrangements (Gurchiek, 2021). The hybrid working model helps in accessing talent pools irrespective of location and its restraints. The hybrid model should be planned out carefully giving premium to the space available for maintaining social distancing, number of employees, mode of communication, and job requirements (Maurer, 2021).

\section{Predictions Regarding the Future}

Based on the current understandings and information gathered from existing literature, the following changes can be predicted:

a. Resourcefulness is the ability to deploy the existing resources in innovative ways and create opportunities. It is one of the key strategies that can be utilized in the New Normal.

b. Flexible work schedules and lead from home or virtual leadership will become the norm.

c. Virtual Employee Assistance Programs
(VEAP), which provides online/ virtual assistance for addressing the psychological, emotional, and physical well-being of the employees, will become an integral component of organizations. Many institutions and start-ups will offer innovative and evidence-based VEAP support services to organizations.

d. Online and digital services will be widespread in the hospitality sector commencing from the room booking stage and ending in the check-out process.

e. Cross-industry temporary employeesharing will be highly valuable in the post-pandemic period.

f. Digitalization, work automation, and new communication technologies will thrive in many industries in the new normal.

g. Self-employed workers will increasingly market their services through digital and social media platforms.

\subsection{Conclusion}

The pandemic created extensive damage in the business sectors and industries. Many industries suffered the havoc, yet some reacted proactively. The situation demanded a paradigm shift in industrial operations and human resource management strategies. Learning from past and present experiences, human resource development should be carried out to meet the vast challenges and explore the opportunities in the new normal.

\section{Acknowledgments}

The authors thank the services of Ms. Dimple Khurana and Mr. Nithin Prakash for editing the manuscript. 


\section{References}

Agarwal, P. (2021). Shattered but smiling: Human resource management and the wellbeing of hotel employees during COVID-19. International Journal of Hospitality Management, 93, 102765. https://doi. org/10.1016/j.ijhm.2020.102765

Altındağ, E., \&Bilaloğlu Aktürk, H. (2020). The impact of new generation management approaches on the firm performance: The moderating role of strategic human resource management applications. SAGE Open, 10(3), 215824402094884. https://doi. org/10.1177/2158244020948845

Armstrong, M., \& Taylor, S. (2014). Armstrong's handbook of human resource management (13thed.). KoganPagen.

Arora, P., \& Suri, D. (2020). Redefining, relooking, redesigning, and reincorporating HRD in the post Covid 19 context and thereafter. Human Resource Development International, 23(4), 438-451. https://doi.org/10.1080/13678868. 2020.1780077

Aurelia, S., \& Momin, M. M. (2020). Global reverberation and prediction for HRM amid and after COVID-19: A technological viewpoint. Materials Today: Proceedings. https://doi.org/10.1016/j.matpr.2020.11.544

Bavik, Y. L., Shaw, J. D., \& Wang, X. H. F. (2020). Social support: Multidisciplinary review, synthesis, and future agenda. Academy of Management Annals, 14(2). https://doi.org/10.5465/ annals.2016.0148
Bhusan, R. \& Verma, P. (2021, April 17). Covid second wave deals another blow to jobs in the hospitality sector. Economic Times. https://economictimes.indiatimes.com/ industry/services/hotels-/-restaurants/covidsecond-wave-deals-another-blow-to-jobs-inhospitality-sector/articleshow/82111265.cms

Borghouts - van de Pas, l., Bosmans, M., \& Freese, C. (2021). Unemployment prevention: The role of human resource management in job-to-job transitions in the event of redundancy. European Journal of Social Security, 23(2), 103-119. https://doi. org/10.1177/1388262721995209

Caballero-Morales, S.-O. (2021). Innovation as recovery strategy for SMEs in emerging economies during the COVID-19 pandemic. Research in International Business and Finance, 57, 101396. https://doi.org/10.1016/j. ribaf.2021.101396

Cooke, F. L., Xiao, Q., \& Xiao, M. (2020). Extending the frontier of research on (strategic) human resource management in China: A review of David Lepak and colleagues' influence and future research direction. The International Journal of Human Resource Management, 32(1), 183-224. https://doi.org/10.1080/0958 5192.2020.1803949

DeLeon, R. \& Geller, J. (2020). Here's however major workforce has been impacted by the coronavirus pandemic. CNBC. https://www. cnbc.com/2020/03/13/workforce-wirecoronavirus-heres-what-every-majorcompany-is-doing-about-the-pandemic.html 
Fachrunnisa, O., \& Hussain, F. K. (2020). Blockchainbased human resourcemanagement practices for mitigating skills and competencies gap in workforce. International Journal of Engineering Business Management, 12, 184797902096640. https://doi.org/10.1177/1847979020966400

Gurchiek, K. (2021, January 27). Hybrid work model likely to be new normal in 2021. Society of Human Resource Management. https://www. shrm.org/hr-today/news/hr-news/pages/ hybrid-work-model-likely-to-be-new-normin-2021.aspx

Hite, L. M., \& McDonald, K. S. (2020). Careers after COVID-19: Challenges and changes. Human Resource Development International, 23(4), 427-437. https://doi.org/10.1080/13678868. 2020.1779576

Howe, D. C., Chauhan, R. S., Soderberg, A. T., \& Buckley, M. R. (2020). Paradigm shifts caused by the COVID-19 pandemic. Organizational Dynamics, 50(4), 100804. https://doi. org/10.1016/j.orgdyn.2020.100804

Kuckertz, A., Brändle, L., Gaudig, A., Hinderer, S., Morales Reyes, C. A., Prochotta, A., Steinbrink, K. M., \& Berger, E. S. C. (2020). Startups in times of crisis - a rapid response to the COVID-19 pandemic. Journal of Business Venturing Insights, 13, e00169. https://doi.org/10.1016/j. jbvi.2020.e00169

Le, D., \& Phi, G. (2021). Strategic responses of the hotel sector to COVID-19: Toward a refined pandemic crisis management framework. International Journal of Hospitality
Management, 94, 102808. https://doi. org/10.1016/j.ijhm.2020.102808

Margherita, A., \& Heikkila, M. (2021). Business continuity in the COVID-19 emergency: A framework of actions undertaken by worldleading companies. Business Horizons, 64(5), 683-695. https://doi.org/10.1016/j. bushor.2021.02.020

Mathew K V, B., \& Walarine, M. T. (2021). Work from home: The new normal. In M.B. Saleem, S. Maganti, P. Gangulay, V.R.R. Gandretti, N. Naleem (Eds.). Role of human resource and customer relationship management in the current scenario (pp. 76-84). Agar Publications. https://www.researchgate.net/ publication/349062812_Work_From_Home_ The_New_Normal

Maurer, R. (2021, February 8). What to consider when moving to a hybrid work model. Society of Human Resource Management. https:// www.shrm.org/hr-today/news/hr-news/ pages/what-to-consider-when-moving-to-ahybrid-work-model.aspx

McLean, G. N., \& Jiantreerangkoo, B. (2020). The role of national HRD in an era of COVID-19. Human Resource Development International, 23(4), 418-426. https://doi.org/10.1080/1367 8868.2020.1780066

Meister, J. (2020, March). The Impact of the Coronavirus on HR and the new normal of work. Forbes. https://www.forbes.com/sites/ jeannemeister/2020/03/31/the-impact-ofthe-coronavirus-on-hr-and-the-new-normalof-work/?sh=3e935a882b60 
Microsoft. (2020). Guide to working from home http [PowerPoint slides]. https://www.microsoft. com $/ \mathrm{microsoft}-365 / \mathrm{blog} / \mathrm{wp}$-content/ uploads/sites/2/2020/04/Work-from-homeguide.pptx

Moher, D., Liberati, A., Tetzlaff, J., \& Altman, D. (2009). Preferred reporting items for systematic reviews and meta-analyses: The PRISMA statement. PLoS Medicine, 6(7), e1000097. https://doi.org/10.1371/journal. pmed.1000097

Pappas, N., \& Glyptou, K. (2021). Accommodation decision-making during the COVID-19 pandemic: Complexity insights from Greece. International Journal of Hospitality Management, 93, 102767. https://doi. org/10.1016/j.ijhm.2020.102767

Rondi, E., Überbacher, R., von Schlenk-Barnsdorf, L., De Massis, A., \& Hülsbeck, M. (2021). One for all, all for one: A mutual gains perspective on HRM and innovation management practices in family firms. Journal of Family Business Strategy, 100394. https://doi.org/10.1016/j. jfbs.2020.100394

Samimi, E., \& Sydow, J. (2021). Human resource management in project-based organizations: revisiting the permanency assumption. The International Journal of Human Resource Management, 32(1), 49-83. https://doi.org/10 $.1080 / 09585192.2020 .1783346$

Senge, P. M. (1997). The fifth discipline. Measuring Business Excellence, 1(3), 46-51. https://doi. org/10.1108/eb025496
Shankar, K. (2020). The Impact of COVID-19 on IT Services Industry - Expected Transformations. British Journal of Management, 31(3), 450-452. https://doi.org/10.1111/1467-8551.12423

Shin, J. C., Li, X., Byun, B. K., \& Nam, I. (2020). Building a coordination system of HRD, research and industry for knowledge and technologydriven economic development in South Asia. International Journal of Educational Development, 74, 102161. https://doi. org/10.1016/j.ijedudev.2020.102161

Shore, L. M., Cleveland, J. N., \& Sanchez, D. (2018). Inclusive workplaces: A review and model. Human Resource Management Review, 28(2), 176-189. https://doi.org/10.1016/j. hrmr.2017.07.003

Society for Human Resource Management. (2021, November 25). Introduction to the human resources discipline of diversity, equity and inclusion. https://www.shrm. org/resourcesandtools/tools-and-samples/ toolkits/pages/introdiversity.aspx

Tan-Torres Edejer, T., Hanssen, O., Mirelman, A., Verboom, P., Lolong, G., Watson, O. J., Boulanger, L. L., \& Soucat, A. (2020). Projected healthcare resource needs for an effective response to COVID-19 in 73 low-income and middle-income countries: A modelling study. The Lancet Global Health, 8(11), e1372e1379. https://doi.org/10.1016/S2214109X(20)30383-1

Tonne, C. (2021). Lessons from the COVID-19 pandemic for accelerating sustainable 
development. Environmental Research, 193, 110482. https://doi.org/10.1016/j. envres.2020.110482

Wang, B., Liu, Y., Qian, J., \& Parker, S. K. (2021). Achieving effective remote working during the COVID-19 pandemic: A work design perspective. Applied Psychology, 70, 16-59. https://doi.org/10.1111/apps.12290 